\title{
A Cooperative Game Theory Approach to Resource Allocation in Wireless ATM Networks
}

\author{
Xinjie Chang ${ }^{l}$ and Krishnappa R. Subramanian \\ Network Technology Research Center, School of EEE, \\ Nanyang Technological University, Singapore 639798 \\ changxjeieee.org \\ esubramentu.edu.sg
}

\begin{abstract}
In the emerging wireless ATM networks, resource allocation with handoff consideration plays an important role in the quality of service (QoS) guarantee for multiple traffic sources. As efficiency is an important performance issue which is most widely used, the concept of fairness should also be considered. In this paper we investigate a fair and efficient resource allocation scheme for two types of traffics contending for the shared network resource. Based on the cooperative game theory, we model the fair and efficient allocation problem as a typical bargaining and arbitration problem, while the issues of efficiency and fairness are considered simultaneously by using the axiom approach. By modeling the corresponding queuing system as a Markov chain and using the Markov decision process (MDP) analysis, we convert the solution of optimal allocation policies into a typical linear programming problem for which the well-known simplex type algorithms can be easily implemented. Simulation results are also provided.
\end{abstract}

\section{Introduction}

Asynchronous Transport Mode (ATM) networks and wireless (cellular) communications networks are merging to form wireless ATM networks. While this integration can provide many benefits, it also leads to many technical challenges in implementation. In the interconnected heterogeneous networks the quality of service (QoS) issue needs to be resolved for different classes of traffic, as the performance criteria differ widely from low speed, less reliable cellular systems, to high speed ATM networks. Since wireless communication systems are bandwidth (network resource) limited, it is important from the network management standpoint to maximize the utility of network resources. In order to provide QoS guarantee for multiple traffic classes in a wireless ATM network, resource allocation is one of the central issues to be studied.

Resource allocation has been extensively studied [2]. Unfortunately, most of the schemes are designed for ATM networks and with the assumption that the terminals are fixed. As smaller cells are always used in wireless communication systems to

\footnotetext{
${ }^{1}$ Xinjie Chang is currently working in the Information \& Telecommunication Technology Center (ITTC), University of Kansas and can be reached at: changxj@ittc.ukans.edu.
} 
meet the need for increased system capacity and spectrum efficiency, the number of cell boundary crossing increases significantly and thus the need for frequent and efficient handoff also increases. As a result, network resource allocations with handoff consideration in wireless ATM networks need to be resolved.

As there are many types of traffics with frequently conflicting performance criteria need to be supported on the limited network resources, multiple objective optimization techniques are needed to get the optimal allocation scheme [11]. Game theory provides a strong mathematical tool for this kind of problem. In early works, attention was mostly focused on the concept of efficiency, such as network resource utilization or throughput maximization. Based on the competitive game theory, the Nash equilibrium solution and Pareto-optimality were used to achieve efficiency. Although this solution can achieve global optimization, it is still shown that there are some cases in which the performance for one or some of the users can be very bad [10]. That is, the performance of some users may be sacrificed to achieve system efficiency, which is unfair to those users. As the quality of service required by various traffic types should be maintained, the concept of fairness is attracting more and more attention. The cooperative game theory provides a strong mathematical tool for the synthesis and analysis of the fair-efficient allocation schemes for the multi-service network [3]. In this paper, we present an optimal resource allocation scheme with handoff priority for wireless ATM networks based on this methodology. We consider two types of traffic to a network node: new calls and handoff calls with higher priority assigned to handoff traffics. By viewing the two types of calls as two players of a cooperative game, we model the problem as a two-person arbitration problem and use the Modified-Thomson scheme to solve optimal solution. Furthermore, by considering the corresponding queuing network as a Markov chain and using the Markov decision process (MDP) analysis, we convert the problem considered into a typical linear programming problem and use the well-known simplex-type algorithm to get the optimal allocation policies for each state. In our analysis, both the efficiency and the fairness features are easily considered by the axiom approach.

This paper is organized as follows: Section 2 provides a brief review of the cooperative game theory, especially the bargaining models and the arbitration schemes. In Section 3, the network model is described and the Modified-Thomson scheme based on the MDP analysis is presented. As an example, some numerical results are provided in Section 4, which illustrate the advantages of our method. Finally, the discussion and the directions for the future work are presented in Section 5 .

\section{Cooperative Game Theory: Bargaining and Arbitration Model}

In the cooperative game, players are assumed to be free to cooperate and bargain to obtain mutual advantage in contrast to the non-cooperative (competitive) game case, in which context communications are not allowed [4]. As bargaining and arbitration are most often used in this kind of problem, we first introduce these two concepts in the following subsections. 


\subsection{Bargaining Problem}

Cooperative games [5] concern at least two players, who may try to reach agreement that can give them mutual advantages. In two-person cooperative game, we can use a region $R$ to denote the problem set and use $(u, v)$ to denote a point in $R$ while $u$ and $v$ represent the two players' utility, respectively. In bargaining situations, the players will act cooperatively to discard all jointly dominated pairs (a pair $(u, v)$ is said to be dominated by another pair $(r, s)$ if both $u \leq r, v \leq s$ )and all undominated pairs which fail to give each of them at least the amount he could be sure of without cooperation.

It is well known that if the players restrict their attention to the bargaining set and bitterly bargain over which point to select, then rational players will frequently fail to reach an agreement [5]. For this case, we can assume that the players will be willing to resolve their conflict through an arbiter--an impartial outsider who will sincerely envisage his mission to be "fair" to both players. Thus, we can use the so-called arbitration schemes to get the optimal allocation solution.

\subsection{Arbitration Scheme}

One of the favorite approaches to get the arbitration solution is the so-called axiomatic approach that exams the subjective intuition of "fairness" and optimality, and formulates these as a set of precise desiderates that any acceptable scheme must fulfill. Once these axioms are formalized, then the original problem is reduced to a mathematical investigation of the existence and characterization of arbitration schemes.

Nash studied this kind of problem in 1950[5] and provided a formal definition and solution. The bargaining problem $\left[R,\left(u^{0}, v^{0}\right)\right]$ is characterized by a region $R$ of the plane and a starting point $\left(u^{0}, v^{0}\right)$, and we can use $F$ to denote an arbitration scheme which maps a typical bargaining game into an arbitrated outcome. Four axioms are defined:

Invariance (inv): The arbitrated value is independent of the unit and origin of the utility measurement (invariance with respect to linear transform).

Independence of irrelevant alternatives (iia) If some of the possible utility combinations were dropped from $R$, resulting a subset $R^{\prime}$, then if $\left(u^{*}, v^{*}\right)$ still left in the $R^{\prime}$, then arbitration value for the new problem will not change.

Efficiency--Pareto-optimality (po): No one can be made better without making the other worse off.

Symmetry: If $R$ is symmetry about the axis $u=v$ and $\left(u^{0}, v^{0}\right)$ is on the axis, then the solution point is also on the axis.

These four axioms fully characterized the Nash solution. Besides Nash's scheme, some alternative solutions schemes have also been reported [5]. By introducing the concept of preference functions, $\mathrm{Cao}[6]$ also formed a mathematical interpretation of the arbitration schemes. 
Definition 1: preference function

Player $i$ 's preference function $v_{i}$ for the outcomes of a bargaining problem $[R,(0,0)]$ is a function of the utilities $u$ and $v$ and the set $R$, i.e., $v_{i}=f_{i}(u, v, R)$, where $(u, v) \in R$.

Note: under the axiom (inv), without losing any generality, we can always linearly transform any pair $\left\lfloor R,\left(u^{0}, v^{0}\right)\right\rfloor$ in such a way that:

$$
\left(\max \left(u^{\prime}: u^{\prime} \in R\right)=1, \max \left(v^{\prime}: v^{\prime} \in R\right)=1\right) \&\left(u^{0}, v^{0}\right)^{\prime}=(0,0)
$$

Which is called normalization.

For our problem, the preference function can be defined as $[3,8]$ :

$$
\begin{aligned}
& v_{1}=u+\gamma(1-v) \\
& v_{2}=v+\gamma(1-u)
\end{aligned}
$$

where $\gamma$ is a weighting factor and $\gamma=-1,0,1$ will correspond to the ModifiedThomson, Nash and Raiffa schemes, respectively. The optimal solution is to maximize the product of player's preference functions:

$$
\left.\left(u^{*}, v^{*}\right)=\underset{(u, v)}{\arg (\max }\left\{v_{1} \cdot v_{2}\right\}\right)
$$

In the Modified-Thomson case, as $\gamma=-1$, then $v 1=v 2$, therefore the problem can be reduced to the maximization of $\mathrm{u}+\mathrm{v}$, which will make the problem much simpler to solve.

\section{Network Model}

In mobile communications systems, there are generally two categories of arrivals to a link (i.e., normal and handoff arrivals). In most cases preference should be given to handoffs over normal arrivals (handoff priority schemes), because the dropping of an existing connection will have more impact on performance than the blocking of a new call. The simplest scheme is fixed channel reservation (i.e., to assign $C_{h}$ channels exclusively for handoff arrivals among the total $C$ channels in a cell) [7]. Being a deterministic priority scheme, it is not efficient, so here we use another scheme described below.

To begin, we focus our attention on only one cell which can carry two types of arrivals: normal arrivals and handoff arrivals. We assume:

Normal arrivals offered to the network can be characterized by a Poisson process with average rate $\lambda_{n}$. The holding time can be modeled by an exponential distribution with mean $\mu_{n}^{-1}$. Bandwidth requirement for normal arrivals is denoted by $d_{n}$.

The handoff arrival is also characterized as a Poisson process with average rate $\lambda_{h}$, exponentially distributed holding time with mean $\mu_{h}^{-1}$ and bandwidth requirement $d_{h}$. We assume here that: $d_{h}>d_{n}$.

The total number of channels that available at the cell is denoted by $N$. At any given instant $t$, let $X(t)=\left\{x_{n}=i, x_{h}=j\right\}$ to denote the state of network, where $x_{n}$ and 
$x_{h}$ are the number of normal arrivals and handoff arrivals current in progress in the cell, respectively.

Therefore, the system state space can be denoted by

$$
E=\left\{(i, j): 0 \leq i, j, 0 \leq i * d_{n}+j * d_{h} \leq N\right\}
$$

and can be divided into several subspaces [7]:

$$
\begin{gathered}
E=E_{i}+E_{b}+E_{e} \\
E_{i}=\left\{(i, j): 0 \leq i, j, 0 \leq i * d_{n}+j * d_{h} \leq N-d_{h}\right\} \\
E_{b}=\left\{(i, j): 0 \leq i, j, N-d_{h}+1 \leq i * d_{n}+j * d_{h} \leq N-1\right\} \\
E_{e}=\left\{(i, j): 0 \leq i, j, i * d_{n}+j * d_{h}=N\right\}
\end{gathered}
$$

If the state is in subspace $E_{e}$, then every coming arrival will be blocked due to lack of channel. In subspace $E_{b}$, only handoff arrival will be blocked due to no channel available and normal will be admitted with some probability. In subspace $E_{i}$, both kinds of arrival can be accepted with some probability. As handoff priority is considered, we assume that the handoff arrivals will always be admitted with probability 1, as long as there are enough channels available and only normal arrivals will be admitted with some probabilities $\alpha(i, j)$, which are the control parameters that need to be calculated.

Based on these assumptions, we can address the problem by using the Markov Decision Process (MDP) model [3,8]. As the state of network is represented by a vector $x$ and the state space $E$. The access policy will be equivalent to an admission subset $A \in E$, and under this policy, an arrival is accepted when the state is $x$ if and only if: $x+e_{k} \in A, \quad e_{k}=\left[\begin{array}{llcll}0 & \ldots & 1 & \ldots & 0\end{array}\right]$. We also can assume that the departure is never blocked. Where the system is in state $x$, reject/accept decision must be made for each coming arrival. Thus, the action space becomes:

$$
B=\left\{\left(a_{n}, a_{h}\right): a_{n} \in\{0,1\}, a_{h} \in\{0,1\} \quad\right\}
$$

The actual action space is a state-dependent subspace of $B$ :

$$
B_{x}=\left\{a \in B, a_{n}=a_{h}=0 \text {, if } x+e_{k} \notin A\right\}
$$

For a MDP model, we can rewrite the state as $X_{B}=\left(x_{n}, x_{h}, x_{\delta}\right)$ with $x_{\delta}$ is a random variable denoting the action of refusal or acceptance for connection of a normal arrival. We can also use a new state space expression: $E_{D}=\left\{(x, a), x \in E, a \in B_{x}\right\}$.

In order to get optimal scheme, we can use the average-reward MDP model, and a reward function $r(x, a)$ should be chosen. We chose the reward function so that optimization objective is to maximize the product of the preference function of normal arrivals and handoff arrivals. Here, we fist choose the Modified-Thomson scheme. Then the problem can be described as a linear programming model:

Max:

$$
Z_{D}=\frac{\lambda_{n} d_{n}}{\mu_{n} N} \sum_{x \in E} p(x, 1)+\frac{\lambda_{h} d_{h}}{\mu_{h} N} \sum_{x \in E_{i}}(p(x, 0)+p(x, 1))
$$

Subject to: 


$$
\begin{gathered}
\sum_{(x, a) \in E_{D}} p(x, a)=1 \\
\sum_{a \in B_{x}} q(x, a, x) p(x, a)=\sum_{\left(x^{\prime}, a^{\prime}\right) \in E_{D}} q\left(x^{\prime}, a^{\prime}, x\right) p\left(x^{\prime}, a^{\prime}\right) \\
p(x, a) \geq 0, \quad \text { for all }(x, a) \in E_{D}
\end{gathered}
$$

Where:

$$
\begin{gathered}
q\left(x^{\prime}, a^{\prime}, x\right)=\left\{\begin{array}{lr}
\lambda_{n} \cdot 1\left\{a^{\prime}=1, x^{\prime} \in E_{i}+E_{b}\right\} & x^{\prime}=\left(x_{n}-1, x_{h}\right) \\
\lambda_{h} \cdot 1\left\{x^{\prime} \in E_{i}\right\} & x^{\prime}=\left(x_{n}, x_{h}-1\right) \\
x_{n}^{\prime} \mu_{n} & x^{\prime}=\left(x_{n}+1, x_{h}\right) \\
x_{h}^{\prime} \mu_{h} & x^{\prime}=\left(x_{n}, x_{h}+1\right) \\
0 & \text { otherwise }
\end{array}\right. \\
q(x, a, x)=\lambda_{1} \cdot 1\left\{a=1, x \in E_{i}+E_{b}\right\}+\lambda_{2} \cdot 1\left\{x \in E_{i}\right\}+x_{1} \mu_{1}+x_{2} \mu_{2}
\end{gathered}
$$

Also, the blocking probability constraints can be included as:

$$
\begin{aligned}
& \sum_{E} p(x, 0) \leq B_{n} \\
& \sum_{E_{b}}(p(x, 0)+p(x, 1))+\sum_{E_{e}} p(x, 0) \leq B_{h}
\end{aligned}
$$

Then, the two-phase simplex algorithm can be used to find the optimal solution. After getting $p(x, d)$, the admission probabilities can be calculated by:

$$
\alpha(i, j)=p(i, j, 1) /(p(i, j, 0)+p(i, j, 1))
$$

\section{Numerical Example}

We constructed a network layer model in the OPNET environment for our simulation scenario, as shown in Figure 1. The network node consists of two traffic source modules, a switch and a sink module. The two traffic source modules generate Poisson type traffics that represent new calls and handoff calls, respectively. The switch is viewed as a finite buffer queue with finite capacity. The sink node is only used for simulation purpose, which can destroy the received packets so that there are enough system resources (memory) for the simulation program. The detailed internal process layer model for the admission control module is shown in Figure 2. In analysis, we assume that the arrival rate of handoff is known a priori. But in fact, the parameter is implicit and must be determined from the process dynamics [6].

Here, the load is defined as:

$$
\mathrm{u}=\mathrm{d}_{\mathrm{n}}\left(\lambda_{\mathrm{n}} / \mu\right)+\mathrm{d}_{\mathrm{h}} *\left(\lambda_{\mathrm{h}} / \mu\right)
$$

By increasing the arrival rate of these two sources (thus the load), we get the loss ratio increased as shown in Figure 3 and 4. Figure 3 gives an example of the variation of loss ratio $b_{1}$ with the increasing of offered load where, for a value of offered load, an optimal control is computed. In the figure, the curve for the case without control is attached for comparison. Figure 4 shows the variation of loss ratio $b_{2}$ against the changing of offered load, the curve for the case without control is also attached for comparison. In Figure 5, the improvement of system utilization against the offered 
load is illustrated by the comparison of the cases without control and with optimal control. The following can be seen:

1) In the case without control, the handoff traffic always has a higher loss ration due to its higher bandwidth requirements.

2) The higher loss ratio of handoff traffic means unfair allocation.

3) In the case with optimal control, we can control the system so that both traffics have performance improvement and thus improvement on the system utilization.

4) Under our optimal control, the two types of traffic not only get performance improvement but also achieve similar performance, which is viewed as a fair allocation of resource.

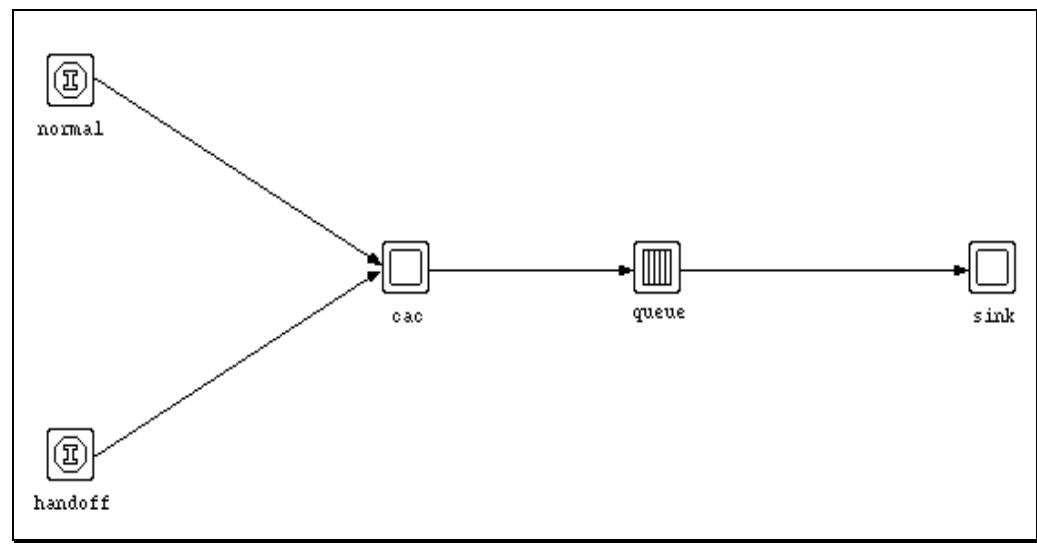

Fig. 1. Node model

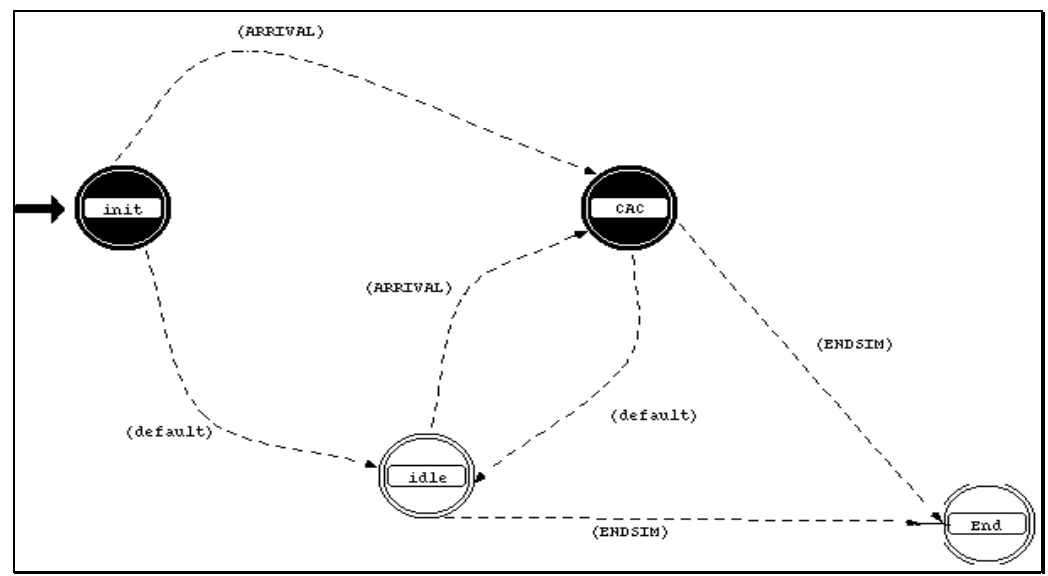

Fig. 2. CAC Process Model 
5) We have to admit that although our scheme can achieve good performance under light and moderate traffic load, there do exist some cases, when the load is very high so that no feasible solution can be obtained. This is the one shortcoming of the scheme.

\section{Conclusion}

A fundamental problem in connection oriented multi-service networks is finding the optimal policy for call acceptance. In this article, we have presented a fair-efficient channel assignment policy based on the cooperative game theory, and especially, the arbitration scheme and axiom approach. Theoretical anaylsis and numeriacl results indicate that:

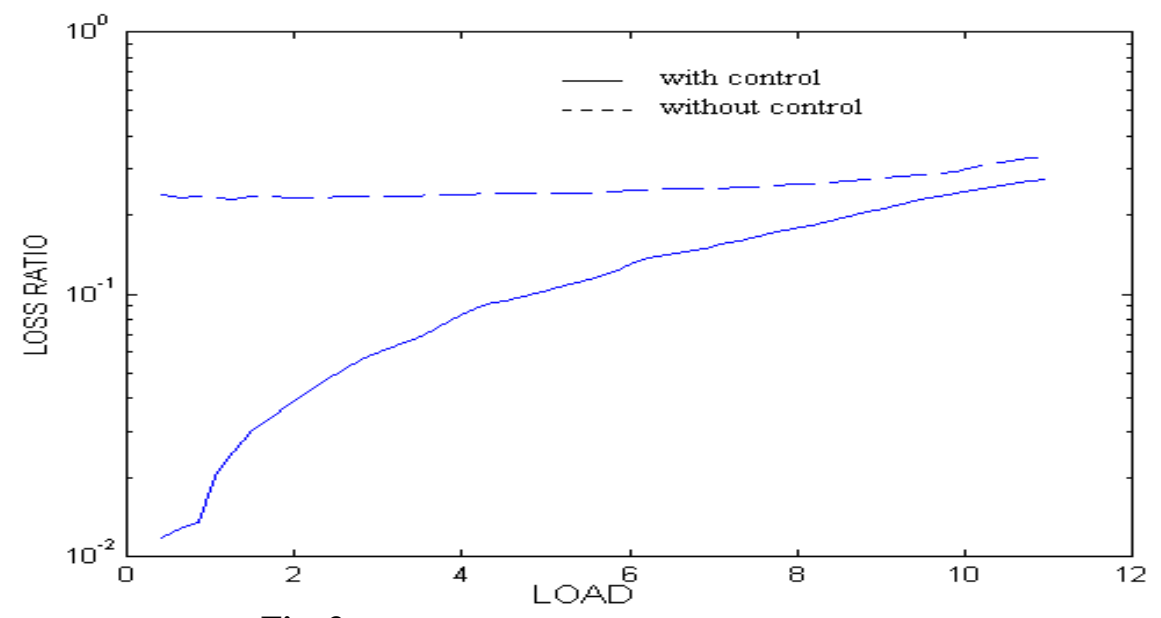

Fig. 3. Loss ratio against load (for handoff calls)

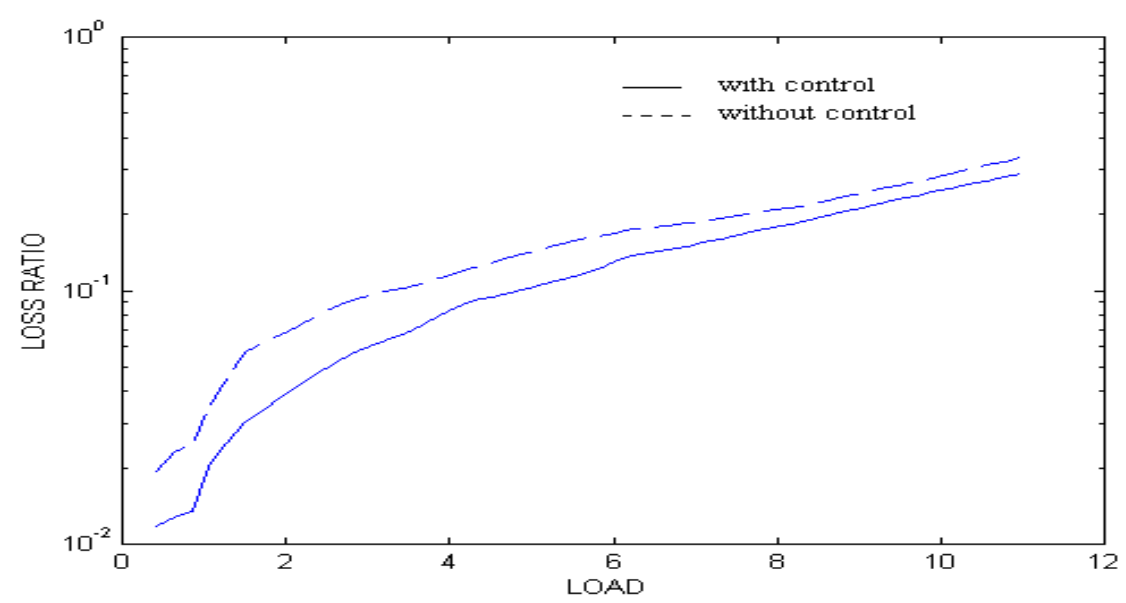

Fig. 4. Loss ratio against load (for new calls) 


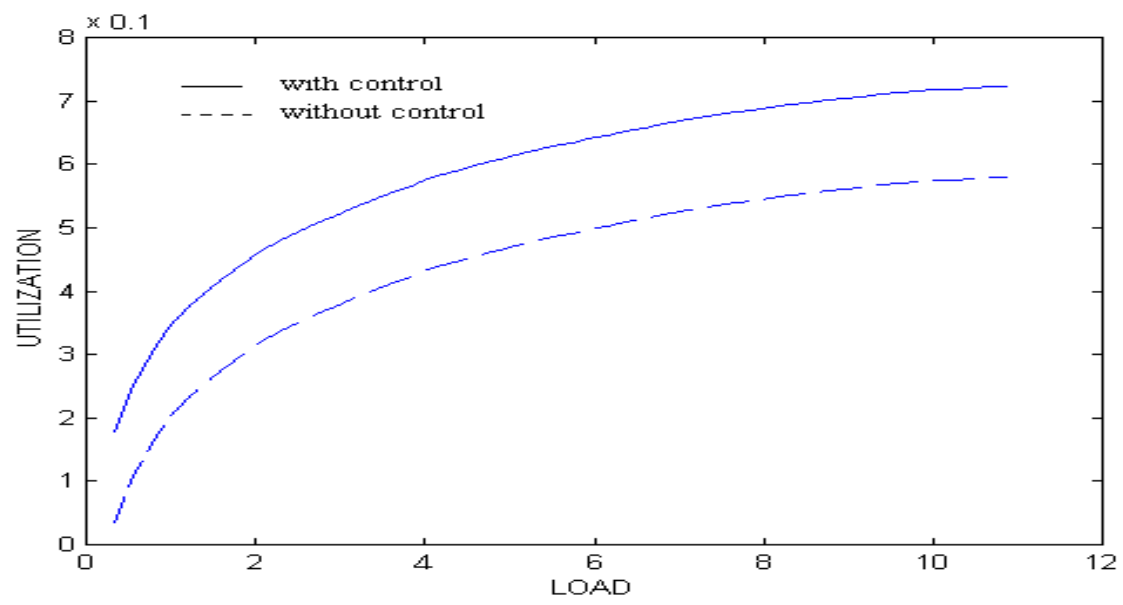

Fig. 5. Utilization improvement

An optimal resource allocation scheme is devised to maximize system utilization while maintaining a fair allocation of resources among competing users, satisfying their individual QoS requirements..

The arbitration scheme and axiom approach are quite appropriate and applicable to resolve these issues

Allocation policy for each state can be calculated by solving a typical linear programming problem while the well-known simplex type algorithms can be used.

After pre-calculation of allocation policies for each state, resource allocation scheme can be implemented on-line very easily and performed at high speed, only a simple table-search is needed.

While the model proposed in this paper can work well for some modest size networks (small state and admission policy spaces), the evaluation of optimal allocation for large-scale networks will become intractable due to the large state and policy spaces. In such cases, some state space reduction and approximation algorithms need to be investigated.

\section{References}

1. G. P.Pollini, “Trends in Handover Design”, IEEE Communications Magazine, Vol. 34, No.3, 1996

2. Xinjie, Chang, K.R.Subramanian, "Call Admission Control Policies for ATM Networks", WmATM'99, June, 1999, Silicon Valley, San Jose, USA,1999

3. Z. Dziong, L. G. Mason, "Fair-Efficient Call Admission Control Policies for Broadband Networks-A Game Theoretic Framework", IEEE/ACM Transactions on Networking, Vol. 4, No.1,1996

4. Alvin E. Roth, "Game-theoretic models of bargaining", Camb. [Cambridge], New York: Cambridge University Press, 1985.

5. Luce R. Duncan, Raiffa, Howard, "Games and Decisions: Introduction and Critical Survey”, New York: Dover Publications, 1989, c1957. 
6. X. Cao, "Preference Function and Bargaining Solution", Proceeding of IEEE CDC, 1982

7. Cong Li, "A Study on Dynamic Resource Allocation and Handoff in Cellular Radio System”, M. Eng. Thesis, School of EEE, Nanyang Technological University, Aug, 1997

8. T. Oda, Yu Watanabe, "Optimal Trunk Reservation for a Group with Multi-slot Traffic Streams", IEEE Transaction On Communications", Vol.38, No.7, 1990

9. OPNET 3.5.A User Manual, MIL3 Company, 1997

10. D. Douligeris, R. Mazumdar, "More On Pareto-Optimal Flow Control", 26 ${ }^{\text {th }}$ Annual Alleton Conference on Communication, Control ad Computing, 1988

11. D. Douligeris, R. Mazumdar, "On Pareto-Optimal Flow Control in a Multiclass Environment", $25^{\text {th }} \quad$ Annual Alleton Conference on Communication, Control ad Computing, 1987 